\title{
Medición de materiales con radiación Terahertz
}

\author{
Measurement of Materials with Terahertz Radiation
}

\author{
A. F. Escobar-Mejía iD
}

\begin{abstract}
Terahertz (THz) radiation or T-Ray defines electromagnetic waves with a frequency range between $0.1 \mathrm{THz}$ and $10 \mathrm{THz}$. It is possible to categorize radiation detection techniques in the THz band in coherent and incoherent techniques. The main difference between them is that in the coherent technique, both the amplitude and the phase of the received signal are determined; but, in the incoherent technique, only the intensity of the signal is measured. THz spectroscopy in the time domain is generally a coherent method. Direct detectors such as pyroelectrics are mainly incoherent detectors. This article presents the experimental measurement of different materials when irradiated with terahertz radiation, applying $\mathrm{THz}$ spectroscopy techniques in the time domain. The radiation is emitted by a photoconductive antenna, excited by a femtosecond laser. The results are obtained by measuring the $\mathrm{THz}$ pulse transmitted through the materials by implementing coherent and incoherent detection systems, analyzing the data obtained, it was possible to obtain the refractive index and absorption coefficient, in case of the coherent technique, and the absorption coefficient in the incoherent technique, for the different materials studied in this work. With these results, it is concluded that it is possible to perform a characterization of different types of materials when irradiated with THz radiation, which leads to various applications from safety to quality control.
\end{abstract}

Index Terms - Absorption coefficient, Photoconductive antennas, Pyroelectric detection, Refraction index, Terahertz radiation.

Resumen - La radiación Terahertz (THz) o Rayos T, define las ondas electromagnéticas con un rango de frecuencias entre 0,1 THz y 10 THz. Es posible categorizar las técnicas de detección de radiación en la banda de los $\mathrm{THz}$ en técnicas coherentes e incoherentes. La principal diferencia entre ellas es que en la técnica coherente se determinan tanto la amplitud como la fase de la señal recibida; pero, en la técnica incoherente, solo se mide la intensidad de la señal.

La espectroscopía THz en el dominio del tiempo es, generalmente un método coherente. Los detectores directos como los piroeléctricos son principalmente detectores incoherentes. Este artículo presenta la medición experimental de diferentes materiales cuando se irradian con radiación de terahertz, aplicando técnicas de espectroscopia $\mathrm{THz}$ en el dominio del tiempo. La radiación es emitida por una antena fotoconductora, excitada por un láser de femtosegundos. Los resultados se obtienen al medir el pulso THz transmitido a través de los materiales al implementar sistemas de detección coherentes e incoherentes, al analizar los datos obtenidos, se logró obtener el índice de refracción y coeficiente de absorción, en caso de la técnica

A. F. Escobar-Mejía. pertenece al Centro de Investigaciones Ópticas, Camino Centenario y 506, CP 1897 CC 3, Manuel B. Gonnet, Buenos Aires, Argentina y la facultad de Ingeniería de la Universidad Nacional de La Plata, coherente, y el coeficiente de absorción en la técnica incoherente, para los distintos materiales estudiados en este trabajo. Con estos resultados se concluye que es posible realizar una caracterización de distintos tipos de materiales al ser irradiados con radiación THz, lo cual conlleva a diversas aplicaciones desde la seguridad hasta el control de calidad.

Palabras claves - Coeficiente de absorción, Antenas fotoconductoras, Detección piroeléctrica, Índice de refracción, Radiación Terahertz.

\section{INTRODUCCIÓN}

La espectroscopia $\mathrm{THz}$ se ha utilizado para estudiar una variedad de fenómenos físicos, desde las transiciones atómicas a la dinámica de las moléculas biológicas, por lo tanto, implica una amplia gama de disciplinas que incluyen física, química, ingeniería, astronomía, biología y medicina.

Las técnicas de espectroscopia $\mathrm{THz}$ son una poderosa herramienta para explorar las propiedades de los materiales que han sido inaccesibles hasta hace poco. La espectroscopia $\mathrm{THz}$ en el dominio de tiempo es una técnica en la que las propiedades de un material se prueban con pulsos cortos de radiación $\mathrm{THz}$. Esta técnica es conocida como detección coherente.

Muchos materiales tienen huellas dactilares espectrales únicas en el rango $\mathrm{THz}$ [1], [2]. Las transiciones rotacionales de moléculas gaseosas dan como resultado líneas de absorción angostas que pueden usarse para identificar la estructura molecular y la presencia de especies particulares en, por ejemplo, la atmósfera de la Tierra y el medio interestelar, [3].

Las técnicas de medición con radiación $\mathrm{THz}$, tienen las ventajas de que esta, es una radiación no ionizante, y las técnicas de medición que se implementan son no destructivas, es decir no alteran la muestra bajo estudio y es segura para los seres vivos.

Este trabajo muestra los estudios y análisis realizados con distintos materiales al ser irradiados con radiación $\mathrm{THz}$ utilizando el método de detección coherente, permitiendo determinar el índice de refracción y la absorbancia en esta banda de frecuencias.

Por otra parte, aplicando técnicas de detección incoherente, se analizan materiales mediante la detección con un sensor piroeléctrico, calculando la absorción para distintos materiales en todo el ancho de banda de los $\mathrm{THz}$ del sistema.

Av. 1 y 47, CP 1900, La Plata, Buenos Aires, Argentina (andrese@ciop.unlp.edu.ar) 


\section{SISTEMAS DE MEDIDA.}

\section{A Método de detección coherente de radiación THz.}

La combinación de una emisión de radiación electromagnética en la banda de los $\mathrm{THz}$, provocada por un pulso láser ultrarrápido y un detector de esta radiación, controlado por una réplica de este pulso sincronizado en el tiempo, permitió el desarrollo de un nuevo método de espectroscopia en el dominio temporal en esta banda de frecuencias, denominado Espectroscopia $\mathrm{THz}$ en el dominio del tiempo (EDT - THz) [4]-[6].

Este método, mide la amplitud y fase del pulso de duración de varios picosegundos. El pulso con esta duración, está formado por muchas frecuencias en la banda de los $\mathrm{THz}$, a las que se puede acceder procesando la señal a través de la transformada de Fourier.

El diagrama esquemático de la Fig. 1, representa el espectrómetro $\mathrm{THz}$ en el dominio del tiempo, empleado en este trabajo. El espectrómetro se basa en la generación y detección de radiación $\mathrm{THz}$, mediante antenas fotoconductoras excitadas por un láser de pulsos ultracortos (laser fs), con una duración de pulsos de los femtosegundos de duración. Una lámina de cuarto de onda en conjunto con un polarizador (Polarizador), es empleado para controlar la potencia del haz que excitará a las antenas fotoconductoras. El haz del láser (traza roja) se divide en dos caminos ópticos mediante un divisor de haz o semiespejo (Divisor), siendo un 60\% del mismo transmitido excitando a la antena fotoconductora emisora de radiación $\mathrm{THz}$ (Emisor) y el $40 \%$ restante es reflejado excitando a la antena fotoconductora detectora de $\mathrm{THz}$ (Detector). Tanto el pulso $\mathrm{THz}$ emitido por el Emisor, como el haz láser que excita a la antena detectora, deben coincidir temporal y espacialmente en la antena. Para lograr esto, se varía el tiempo de llegada relativo entre los dos caminos ópticos que excitan a las antenas fotoconductoras. Esto se logra variando la longitud física de uno de ellos, mediante el uso de una etapa de traslación mecánica (Retardo). La antena fotoconductora emisora se polariza mediante una fuente regulada (Fuente), que excita a un modulador de voltaje (Modulador) a una frecuencia determinada, lo cual permite una detección sincrónica sensible a la fase en la adquisición y procesamiento de la señal. La señal es procesada en el bloque Adquisición y Procesamiento de la señal, marcado con líneas de punto y raya en la Fig. 1. Este está compuesto del Amplificador Lock - In (ALI) y una computadora (PC). La antena fotoconductora detectora, produce una corriente proporcional al campo eléctrico instantáneo generado por el pulso de los THz. La corriente generada es registrada por el ALI, el cual procesa la señal diferenciándola del ruido, la PC controla el sistema y almacena los datos para luego ser procesados mediante softwares específicos fuera de línea.

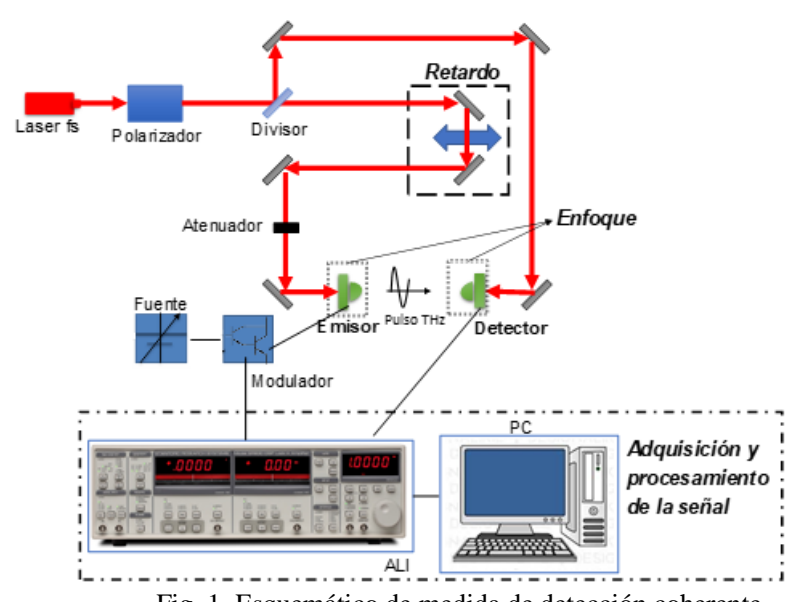

Fig. 1. Esquemático de medida de detección coherente.

\section{B. Método de detección incoherente de radiación $\mathrm{THz}$}

Se desarrolló un sistema de medida donde se registró la intensidad del pulso $\mathrm{THz}$ emitido por la antena fotoconductora en todo su ancho de banda, utilizando un detector piroeléctrico, generando así un sistema de detección incoherente de radiación en la banda de los THz.

A pesar de la gran variedad de detectores a temperatura ambiente, los detectores piroeléctricos son los más empleados, en distintas bandas del espectro electromagnético. Estos ofrecen amplio rango dinámico, gran ancho de banda de detección y altos niveles de sensibilidad [7].

En la Fig. 2 se esquematiza el sistema de detección incoherente de detección de radiación $\mathrm{THz}$, diseñado durante el análisis de materiales.

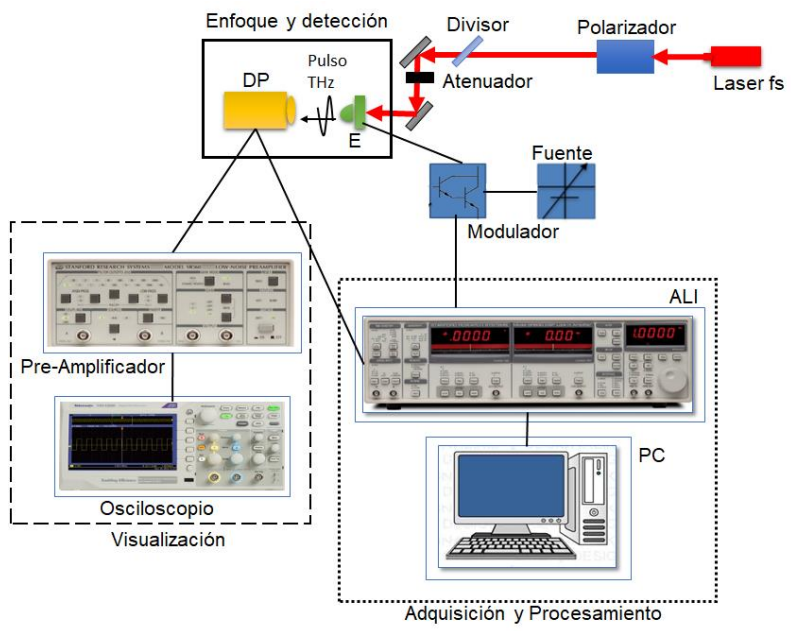

Fig. 2 Esquema de medida de detección incoherente.

El sistema implementado en partes es similar al empleado en detección coherente, empleando la antena fotoconductora con el láser de los femtosegundos para la generación de la señal de los $\mathrm{THz}$, con un polarizador y modulador con los mismos objetivos. La detección es sincrónica se realiza a través del sistema de Adquisición y Procesamiento de la señal (Fig. 2).

\section{ANÁLISIS DE MATERIALES CON DETECCIÓN COHERENTE.}

Para este análisis se seleccionaron materiales que, según la literatura [1], [4], [5], tuvieran distintas respuestas al ser 
irradiados con radiación THz. Los materiales analizados se muestran en la tabla I, con su respectivo espesor.

TABLA I.

MATERIALES ANALIZADOS.

\begin{tabular}{cc}
\hline \hline Material & Espesor $(\mathrm{d})[\mathrm{mm}]$ \\
\hline Silicio monocristalino & 0,5 \\
Silicio orientación (100) & 1,1 \\
Vidrio & 1,0 \\
Papel & 0,1 \\
Polietileno de baja densidad & 0,2 \\
Cartón & 1,2 \\
Goma EVA & 2,0 \\
Madera & 3,7 \\
\hline \hline
\end{tabular}

El sistema utilizado para la medida de materiales con detección coherente es el observado en la Fig. 1. El material se coloca entre la antena emisora y la receptora utilizando un porta objeto y a través del sistema de Adquisición y Procesamiento la señal es recibida. En primer lugar, en la Fig. 3, se comparan tres compuestos de silicio: silicio monocristalino (trazo amarillo oscuro) de $0.5 \mathrm{~mm}$ de espesor, silicio con orientación cristalina 100 (trazo azul) de $1.1 \mathrm{~mm}$ de espesor y vidrio (trazo gris oscuro) de $1 \mathrm{~mm}$ de espesor. Como se observa en la Fig., la radiación $\mathrm{THz}$ será transmitida en mayor o menor medida dependiendo del tipo de silicio presente en la muestra, notándose que, para el silicio monocristalino, la radiación no es transmitida, en cambio para el silicio con orientación cristalina 100 la radiación es transmitida pero fuertemente atenuada, y en el caso del vidrio pyrex se presenta una buena transmisión de radiación THz. Los valores de los picos máximos para los materiales ensayados se observan en la Tabla II.

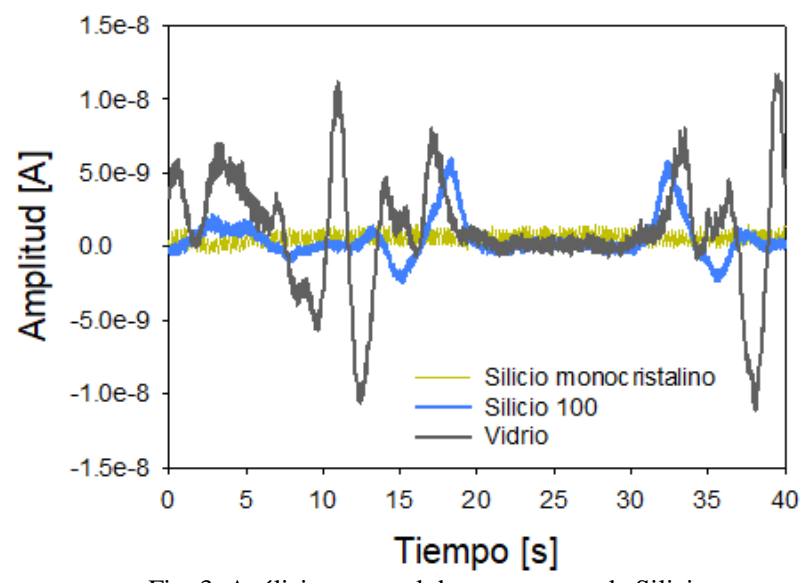

Fig. 3. Análisis temporal de compuestos de Silicio.

Finalmente, en la Fig. 4 se analizan materiales poliméricos, como lo son el papel (trazo negro) de $0.1 \mathrm{~mm}$ de espesor, el polietileno de baja densidad (trazo cian) de $0.2 \mathrm{~mm}$ de espesor, una lámina de cartón (trazo naranja) de $1.2 \mathrm{~mm}$ de espesor, una lámina de etil-vinil-acetato (goma EVA, trazo verde) de $2 \mathrm{~mm}$ de espesor y una lámina de madera (trazo marrón) de $3.7 \mathrm{~mm}$ de espesor. Como se observa en la Fig. 4, y midiendo la amplitud de los respectivos pulsos, los materiales poliméricos se caracterizan por ser buenos transmisores de radiación $\mathrm{THz}$, lo que cambia en este caso, es el nivel de continua entre estos, por eso, esta tecnología es aplicable a sistemas de seguridad, ya que, es posible detectar objetos que estén por ejemplo en cajas de cartón o en sobres de papel.

En la Tabla II se observan los picos máximos de los materiales que serán utilizados para determinar sus características.

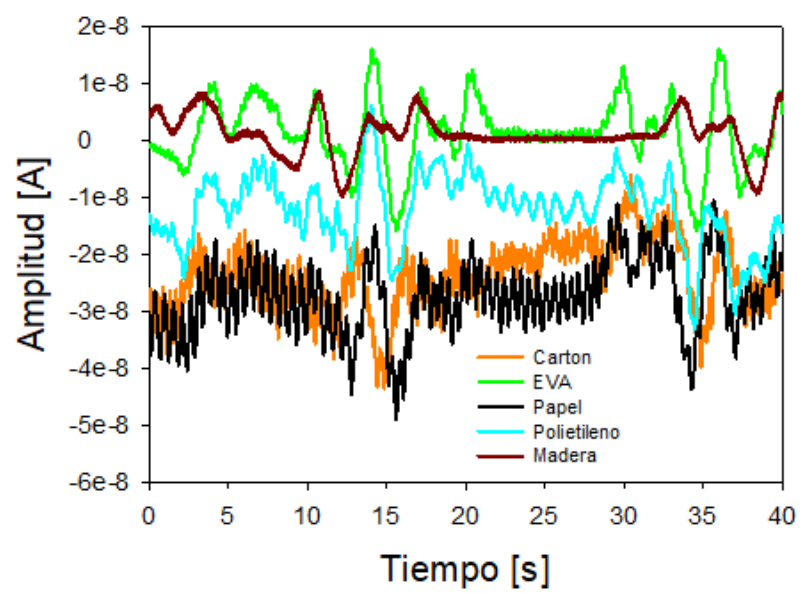

Fig. 4. Análisis de temporal de polímeros.

Para calcular el índice de refracción y la absorbancia de los materiales a través del uso de los datos temporales, se utiliza la información del retardo provocado en el pulso temporal cuando hay muestra presente, y cuando no la hay (sin muestra). A través del retardo generado entre los pulsos se calcula el índice de refracción (n) [1]:

$$
n=1+\frac{c t_{d}}{d}
$$

donde $t d$ es el retardo entre los picos de los pulsos y $d$ es el espesor de la muestra. Esto es válido suponiendo el índice de refracción constante en el ancho de banda del experimento. A partir de los valores pico de los campos eléctricos, de la muestra $(E m)$ y del pulso de referencia (Eref), se puede calcular el coeficiente de absorción de intensidad $\alpha$ si se consideran las pérdidas de Fresnel en ambas caras de la muestra [8]:

$$
\alpha=\frac{2}{d} \ln \left(\frac{4 n}{(n+1)^{2}} \text { Em/Eref }\right)
$$

En la Tabla II, se presentan los resultados de obtener los parámetros ópticos de los materiales analizados mediante la información obtenida en el dominio temporal.

TABLA II.

ÍNDICE DE REFRACCIÓN (N) Y ABSORBANCIA (A) CALCULADO PARA DISTINTOS MATERIALES.

\begin{tabular}{cccc}
\hline \hline Material & tpico [ps] & $\mathrm{n}$ & $\alpha[\mathrm{mm}-1]$ \\
\hline Sin muestra & 14,3622 & & \\
Silicio & 11,6724 & 2,022 & 15,9019 \\
Silicio 100 & 10,1443 & 2,022 & 2,3296 \\
Vidrio & 14,2898 & 2,009 & 0,5748 \\
Papel & 14,0053 & 1,217 & $-2,5096$ \\
Polietileno & 16,0650 & 1,535 & $-1,8682$ \\
Cartón & 18,1101 & 1,332 & $-0,1881$ \\
EVA & 10,9978 & 1,043 & 0,0667 \\
Madera & 6,2589 & 1,314 & 0,3212 \\
\hline \hline
\end{tabular}




\section{ANÁLISIS DE MATERIALES POR CON DETECCIÓN INCOHERENTE.}

Utilizando el sistema de detección incoherente de medida de radiación de los Terahertz como se observa en la Fig. 2, se analizarán los materiales empleados con el método coherente además del dióxido de Zirconio. En este caso se estudia el comportamiento de dichos materiales en todo el ancho de banda de $\mathrm{THz}$ de respuesta del sistema. Los materiales analizados se sitúan a una distancia fija entre la antena fotoconductora y el detector piroeléctrico. Se determina la transmitancia para los materiales analizados, en la banda de frecuencia de los THz. El coeficiente de extinción de cada material [1] se determina como en la ecuación 3:

$$
\alpha=\frac{-\ln \left(\frac{P}{P_{o}}\right)}{d}
$$

La tabla III muestra los materiales analizados y los resultados de aplicar la ecuación (3), donde LDPE corresponde a Polietileno de baja densidad y $\mathrm{ZrO} 2$ es el dióxido de Zirconio.

TABLA III.

MATERIALES IRRADIADOS CON THZ, DETECTADOS CON EL PIROELÉCTRICO.

\begin{tabular}{cccc}
\hline \hline Material & Espesor $[\mathrm{mm}]$ & Potencia detectada $[\mathrm{nW}]$ & $\alpha\left[\mathrm{mm}^{-1}\right]$ \\
\hline $\begin{array}{c}\text { Sin Muestra } \\
\text { Silicio } \\
\text { dopado }\end{array}$ & - & 49,0 & -- \\
Madera & 0,50 & 13,1 & 2,63 \\
LDPE & 1,50 & 6,5 & 1,24 \\
ZrO2 & 0,20 & 42,2 & 0,75 \\
Cartón & 2,00 & 18,2 & 0,50 \\
Goma EVA & 1,20 & 26,6 & 0,48 \\
Papel & 1,54 & 41,7 & 0,10 \\
\hline \hline
\end{tabular}

\section{CONCLUSIONES}

Como se observa en la Tabla II para análisis de materiales con detección coherente, los materiales con baja transmisión de radiación $\mathrm{THz}$ como los metales, tienen coeficientes de absorción más altos que los materiales que presentan alta transmisión como los polímeros. Un caso interesante lo presenta el silicio, ya que, dependiendo del tipo de silicio, se presenta distintas respuestas a la radiación $\mathrm{THz}$, las lentes que componen a las antenas fotoconductoras están fabricadas de silicio de alta densidad, el cual tiene una alta transmisión de radiación $\mathrm{THz}$.

Para los análisis realizados con el método de detección incoherente vemos que al igual que en el caso de la medición coherente, como se observa en la Tabla III, los materiales poliméricos (polietileno, papel, goma EVA, cartón) son muy buenos trasmisores de radiación $\mathrm{THz}$, mientras que en el otro extremo se encuentran los compuestos de silicio (silicio, vidrio). Vale mencionar que el análisis con sistema incoherente permite evaluar las respuestas de los materiales en todo el ancho de banda de los THz utilizado por el sistema, lo cual no ha sido registrado en la literatura.

\section{AGRADECIMIENTOS}

El autor agradece la colaboración del Dr. Jorge O. Tocho y el Dr. J. Alberto Bava, y el Proyecto de Investigación Científica y Tecnología (PICT) número 1550 del Ministerio de Ciencia, Tecnología e Innovación Productiva de Argentina, por el apoyo necesario para la concreción de esta investigación.

\section{REFERENCIAS}

[1] A. K. R. E. Miles, X. -C. Zhang, H. Eisele, Ed., Terahertz frequency Detection and Identification of Materials and Objects, Washington D. C, USA, Springer 2006.

[2] P. C. Upadhya, Y. C. Shen, A. G. Davies and E. H. Linfield, "Terahertz time-domain spectroscopy of glucose and uric acid," J. Biol. Phys., vol. 29, no. 2-3, pp. 117-121, 2003, DOI: 10.1023/A:1024476322147.

[3] C. K. Walker, Terahertz Astronomy, Boca Ratón, FL, USA, CRC Press, 2016.

[4] X. C. Zhang and J. Xu, Introduction to THz wave photonics, New York, NY, USA Springer, 2010.

[5] Y. S. Lee, Principles of terahertz science and technology, New York, NY, USA, Springer 2009.

[6] P. U. Jepsen, D. G. Cooke and M. Koch, "Terahertz spectroscopy and imaging - Modern techniques and applications," Laser Photonics Rev., vol. 5, no. 1, pp. 124-166, 2011, DOI: $10.1002 /$ lpor.201000011.

[7] C. W. Berry, N. Yardimci, and M. Jarrahi, "Responsivity calibration of pyroelectric terahertz detectors", arXiv, Cornell University, 2014, arXiv:1412.6878 [physics.ins-det].

[8] J. O. Tocho and F. Sanjuan, "Optical properties of silicon, sapphire, silica and glass in the Terahertz range," Lat. Am. Opt. Photonics Tech. Dig., no. 3, p. LT4C.1, 2012. DOI: 10.1364/LAOP.2012.LT4C.1

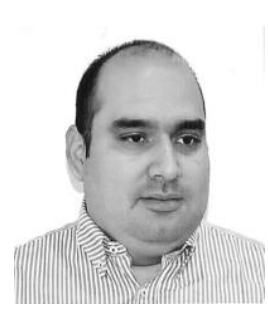

Andrés Felipe Escobar Mejía. (S'86) Ingeniero Físico de la Universidad Tecnológica de Pereira, título recibido en diciembre de 2010. Estudiante de Doctorado en Ingeniería de la Universidad Nacional de La Plata, Argentina, con lugar de trabajo en el Centro de Investigaciones Ópticas de la misma cuidad, desde septiembre de 2011, con beca del Consejo

Nacional de Investigaciones Científicas y Técnicas (CONICET) del Ministerio de Ciencia, Tecnología e Innovación Productiva de Argentina.

ORCID: http://orcid.org/0000-0002-7649-2377 